\title{
Determinants of stability in the perception of subjective contours
}

\author{
CYNTHIA A. LAURIE \\ Otterbein College, Westerville, Ohio \\ and \\ JOEL S. WARM, WILLIAM N. DEMBER, and ROBERT A. FRANK \\ University of Cincinnati, Cincinnati, Ohio
}

\begin{abstract}
The present study constituted an initial experimental effort to examine the fragmentation characteristics of subjective contours within the photopic and upper scotopic ranges of illumination. Four stimulus factors known to influence the visibility of subjective contours-target luminance, inducing area size and contrast, and contour orientation-were examined. Results indicated that subjective contours are indeed unstable perceptual phenomena. On the average, fragmentation or fading occurred after only $15 \mathrm{sec}$ of observation, and some form of stimulus outage was present for $28 \%$ of the viewing time of each stimulus. Fragmentation latency was significantly shorter and total time in fragmentation longer for diamond than for square contours, and total time in fragmentation varied inversely with inducing-area size. Fragmentation tended to occur in whole units rather than in isolated elements, a result reminiscent of the fading of real contours under impoverished viewing conditions.
\end{abstract}

The terms subjective or illusory contours refer to the appearance of edges in areas of the visual field that lack local discontinuities in luminance or wavelength. Examples of a subjective square and diamond are presented in Figure 1.

Although not physically present in the viewing field, subjective contours are psychologically meaningful, and they share many of the functional properties of real figures. For example, geometric illusions and reversible figures can be perceived with subjective contours, they are subject to figural aftereffects, they can be placed in apparent motion, and they are susceptible to kinetic depth information (cf. Warm, Dember, Padich, Beckner, \& Jones, 1987).

Although their functional similarity to real contours is often striking, subjective contours are nevertheless unstable perceptual entities that tend to break up and fade away with prolonged inspection under normal viewing conditions. This aspect of illusory contours can be experienced by gazing continuously at one of the subjective forms in Figure 1 for several seconds. The form, which may appear quite vivid at first, will fade in and out, fragment or disappear entirely, leaving only the open jaws of its "pac man" inducers.

The instability of subjective contours was noted in their initial presentation by Schumann (1900/1987) and has

Portions of this paper were presented at the meeting of the Psychonomic Society, New Orleans, November 1990. Correspondence should be addressed to C. A. Laurie, Department of Psychology, Otterbein College, Westerville, $\mathrm{OH} 43081$.

-Accepted by previous editor, Charles W. Eriksen
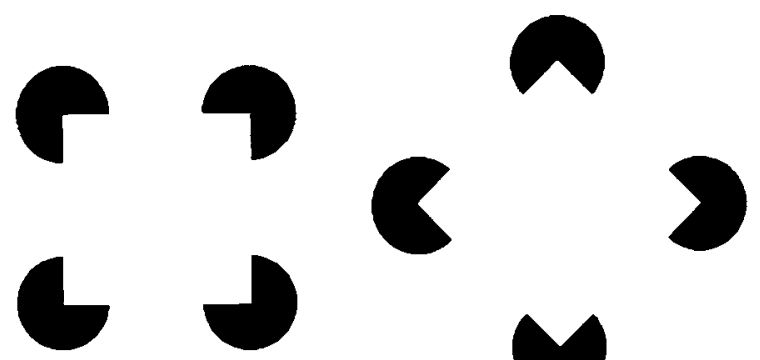

Figure 1. A subjective square and diamond.

been commented upon frequently by others (Coren, 1972; Dumais \& Bradley, 1976; Kanizsa, 1976). However, very little research has been directed toward a systematic examination of the factors controlling it.

To date, the only experimental attack on this problem has been conducted by Halpern and Warm $(1980,1984)$. These investigators made use of very dim electroluminescent viewing conditions, first described by McKinney $(1963,1966)$, which render perceptual experience generally unstable. In this way, they could compare the fragmentation characteristics of subjective and real contours. Halpern and Warm (1980) found that under these viewing conditions, subjective contours were more unstable than their real figure analogues, and that the nature of the fragmentation differed between the two types of figures. Real figures disappeared entirely or portions of them frag- 
mented. For subjective contours, disappearances occurred primarily in the physically present inducing areas, while the subjective edges remained visible, and there were fewer whole figure disappearances. In a later study, Halpern and Warm (1984) demonstrated that subjective contours could be rendered more stable than real contours under dichoptic viewing conditions (different portions of a figure are presented to each eye) wherein figure completion must be accomplished postretinally.

The studies of Halpern and Warm $(1980,1984)$ reveal some of the characteristics of fragmentation in illusory figures and, given the outcome of the dichoptic experiment, suggest that fragmentation of subjective contours may be more centrally determined than the fragmentation of real contours. Note, however, that these studies were conducted under extremely impoverished, scotopic viewing conditions, whereas the bulk of the research on subjective contours has been conducted under richer, photopic conditions. There are no data on the fragmentation characteristics of photopically illuminated subjective contours. To provide such data, we tested the effects of four stimulus factors known to have a strong influence on the perceptual salience of subjective contours: target luminance, inducing-area contrast, inducing-area size, and contour orientation. Several studies have shown that contour clarity and depth stratification vary inversely with target luminance (Bradley \& Dumais, 1984; Parks \& Marks, 1983, 1985). In addition, contour clarity and apparent brightness vary directly with the contrast between inducing areas and their background (Warm et al., 1987), and the apparent brightness of subjective figures also varies directly with the size of their inducing areas (Brigner \& Gallagher, 1974). Moreover, the visibility of subjective contours is enhanced when the contours lie along cardinal as opposed to oblique coordinates (Vogels \& Orban, 1987), a result reminiscent of the oblique effect in the perception of real contours (Appelle, 1972). Thus, in the present study, we explored the fragmentation characteristics of subjective contours by determining whether factors known to influence their perceptual salience also affect their stability.

\section{METHOD}

\section{Subjects}

Ninety-six students from introductory classes at the University of Cincinnati served as subjects. All had normal or corrected-tonormal vision.

\section{Design}

A mixed design was used. Two levels of inducing-area contrast (black $-8 \%$ reflectance; gray $-39 \%$ reflectance) and three levels of inducing-area size (15-, 20-, 25-mm radii) were combined factorially with two contour orientations (cardinal and oblique) and four levels of target luminance $\left(4,2,1,-1 \log _{e} \mathrm{~cd} / \mathrm{m}^{2}\right)$ to produce 48 experimental conditions. Inducing-area contrast and inducingarea size were between-subjects factors; contour orientation and target luminance were within-subjects factors.

\section{Stimuli}

Subjective figures were constructed by drawing black and gray inducing areas on white posterboard. Stimuli with cardinal and oblique contour orientations were generated by arraying the inducing areas to produce square and diamond forms, as is illustrated in Figure 1 . In this way, contour orientation could be varied within the context of a meaningful form while other potentially important elements of the form such as size, number of sides and angles, and the variance of the angles were held constant. In all cases, the diameter of the subjective contour portion of the stimulus array was $5 \mathrm{~cm}$. A small red fixation dot $(1 \mathrm{~mm})$ was placed in the center of all stimuli.

\section{Procedure}

Sixteen subjects were assigned at random to each of the 6 orthogonal combinations of inducing contrast and size. Within each of these conditions, the order in which subjects experienced each of the four levels of target luminance as they proceeded through the experimental session was counterbalanced by means of a Latin square. The sequence in which the subjects viewed the square and diamond forms was balanced within each order of the Latin square.

The stimuli were presented in a black viewing box in an otherwise darkened laboratory. The apparatus was mounted at eye level in front of the seated subject. A port in the box permitted binocular inspection of the stimuli, which were mounted on a frame at the opposite end of the box at a distance of $1.5 \mathrm{~m}$ from the subject's eyes. A reduction screen located inside the box at a distance of $1.3 \mathrm{~m}$ from the subject's eyes provided a uniform field of view that was $3.81^{\circ}$ square. The stimuli were centered within this field. In all cases, the square or the diamond portions of the stimulus array subtended a visual angle of $2.4^{\circ}$. Between trials, the viewing field was shielded by a lighted shutter with a luminance of approximately $1.0 \mathrm{~cd} / \mathrm{m}^{2}$.

The viewing field was illuminated by a 75-W GE soft white bulb in a specially designed lamp mounted on the roof of the viewing box out of the subject's line of sight. Thus, when observing the stimuli, the subject was not aware of the source of illumination. Munsell neutral density filters placed in a holder in front of the lamp permitted the lighting of the visual field to be varied widely without changes in color temperature. The lighting arrangement permitted the subjects to view the stimuli directly without looking through the filters.

The subjects were dark adapted for $5 \mathrm{~min}$ at the beginning of each session under conditions in which the laboratory was illuminated by a dim red light. During this time, the subjects received instructions for the task. They were presented with two geometric forms composed of subjective edges (square and diamond) and were asked to identify them. All subjects were able to do so successfully. They were then told that with prolonged inspection, subjective forms may sometimes fade or fragment. Fragmentation was described as a disappearance or breaking up of the stimulus, either as a whole or in parts. The subjects were instructed to depress a response switch held in their preferred hand as soon as any portion of the stimulus disappeared and to keep the switch depressed until it reappeared. If there was a change in the nature of the fragmentation, the subjects were told to release the switch momentarily and then to depress it again, and to continue to keep it depressed for the duration of the new fragment. The subjects were also instructed that fragmentation is not a universal occurrence and that they should only press the switch in the presence of fragmentation.

The subjects were instructed at the start of each trial to place their chin in the chinrest and to fixate the dot in the center of the subjective form and to maintain that fixation for the duration of the trial. Each stimulus was presented for a 2-min inspection period, which was followed by a 1-min break. The break time was used to permit subjects to indicate the nature of the fragmentations that they had experienced during the trial. Toward that end, the subjects were provided with lap-board mounted sheets of paper containing dotted configurations that matched the figure just seen. For example, if a subject had inspected a diamond, the answer sheet contained 10 dotted diamonds. The subject's task was to trace over the portion of the figure that had disappeared (including whole-figure dis- 
appearances as well as partial or complete disappearances of any given edge) for as many of the kinds of disappearances as could be remembered. The tracing method of report has been shown to be preferable to verbal reports, which bias subjects' responses toward easy verbal descriptions (Halpern \& Warm, 1980; Schuck, 1973). In order to maintain a general level of dark adaptation, a red light adjacent to the subject was used to illuminate answer sheets during the drawing phases of the experiment. The experimenter initiated all trials by depressing the keyboard of an Apple IIe microcomputer, which, in turn, recorded the frequency and duration of the subject's buttonpress responses. The experimental session lasted for approximately $60 \mathrm{~min}$.

\section{RESULTS}

\section{Quantitative Data}

Two measures of stability were determined from the data of each subject on each trial. They included fragmentation latency - the time to first fragmentation-and total time in fragmentation-the time during which the subject reported the stimulus to be completely out of view or partially fragmented.

Time to first fragmentation. Preliminary inspection of the data for individual subjects generally revealed no systematic differences between the first and second presentations of the cardinal-oriented (square) or obliqueoriented (diamond) stimuli under any conditions of luminance, size, or contrast. Thus, the time to first fragmentation was determined by averaging the scores for the two iterations of the square or diamond forms.

Mean times to first fragmentation were computed for all experimental conditions. Inspection of the data revealed that fragmentation occurred rapidly, after only $15 \mathrm{sec}$ of observation on the average. Time to first fragmentation scores were subjected to a 2 (inducing-area contrast) $\times$ 2 (contour orientation) $\times 3$ (inducing-area size) $\times 4$ (luminance) analysis of variance. Perceptual instability was greatest for oblique (diamond, $M=13.5 \mathrm{sec}$ to first fragmentation) as opposed to cardinal (square, $M=15.9 \mathrm{sec}$ to first fragmentation) contour orientations $[F(1,90)=$ $13.16, p<.01]$. All the remaining sources of variance in the analysis failed to reach significance.

Total time in fragmentation. Preliminary inspection of the data for the total time in fragmentation revealed no systematic differences between the first and second iterations of a given contour orientation in any of the combinations of inducing area contrast and size and target luminance. Accordingly, total time values were determined by summing over the two iterations of each orientation condition. Thus, the total observation time on which the data are based is $240 \mathrm{sec}$. Perceptual fading was substantial in all experimental conditions, ranging from 47.8 to $103.9 \mathrm{sec}$ of the 240 -sec inspection period, with an overall mean of $68 \mathrm{sec}$. On the average, therefore, some form of stimulus outage was present during $28 \%$ of the viewing time. The data were subjected to an analysis of variance, which indicated that orientation and inducing area size were the principal factors influencing total time in fragmentation. Fragmentation times were significantly greater for diamonds $(M=72.4 \mathrm{sec})$ than for squares
$(M=63.4)[F(1,90)=17.74, p<.01]$, and total time in fragmentation varied inversely with inducing area size [ $M \mathrm{~s}$ in the 15-, 20-, and 25-mm inducing-area radii conditions were $80.8,68.5$, and 54.5 , respectively, $F(2,90)=4.42, p<.01]$. The effects of the contrast and luminance factors on total time in fragmentation were minimal and were manifested only in complex interactions involving luminance $\times$ size $[F(6,270)=4.19, p<.05]$ and luminance $x$ inducing-area contrast $\times$ contour orientation $[F(3,270)=5.21, p<.01]$.

\section{Qualitative Data}

Measures of fragmentation time provide no insight into the kinds of fragmentations that the subjects experienced or the pattern of the fading. To obtain such information, the subjects' tracings were examined for the presence of the following disappearance categories: whole figure, in which the inducing areas remain visible and fixed in place; single edge; multiple edge (two or more); and other. The last category included drawings that could not fit exclusively into one of the other three, such as whole-figure disappearance in which the "pac man" inducing areas moved outward away from the form or imploded inward toward the center of the form to produce a "clover." It is of interest to note that in the case of the multiple-edge category, disappearances occurred in units, such as parallel sides fading out. In both the single-edge and multipleedge categories, individual lines almost always faded in their entirety.

Within each experimental condition, the frequency of report of any disappearance category was determined by noting the number of subjects (out of $n=16$ ) who drew at least one instance of that category. In order to assess the statistical significance of these results, mean values for each disappearance category were determined by averaging across all experimental conditions. These values and their $5 \%$ fiducial limits were: whole figure, $91 \% \pm 2 \%$; multiple edge, $77 \% \pm 4 \%$; single edge, $45 \% \pm 4 \%$; and other, $37 \% \pm 4 \%$. It is clear from these values that wholefigure disappearances were experienced significantly more often than multiple-edge disappearances, which, in turn, occurred more frequently than disappearances in either the single-edge or the other category, and that disappearances falling in the latter two categories occurred with equal frequency.

\section{DISCUSSION}

The results clearly show that subjective contours are indeed unstable perceptual phenomena. On the average, fragmentation or fading occurred within $15 \mathrm{sec}$ of the onset of a trial, and some form of stimulus outage was present during $28 \%$ of the viewing time. Moreover, fragmentation is controlled by some of the same factors that determine the salience or vividness of subjective figures. Contour stability, for example, was found to vary directly with inducing-area size. This relation is reminiscent of results showing that the perceived sharpness of subjective contours varies directly with the size of their induc- 
ing area (Brigner \& Gallagher, 1974; Petry, Harbeck, Conway, \& Levey, 1983).

Perceptual stability was also enhanced when contour orientation fell along cardinal orientations, forming a square, as opposed to oblique coordinates, forming a diamond. This result is consistent with fading effects obtained with real contours under stabilized image (in the form of an afterimage) conditions (Atkinson, 1972) and with reports indicating that cardinal orientation enhances the general perceptibility of subjective contours (Vogels \& Orban, 1987).

Taken together, these findings suggest that what makes subjective contours visible also makes them more stable. There are some exceptions, however. Inducing-area contrast and target luminance, both previously found to have a strong influence upon the salience of subjective contours (Brigner \& Gallagher, 1974; Gregory, 1977; Jory \& Day, 1979; Warm et al., 1987), had only minimal effects upon perceptual stability. The absence of strong luminance effects was surprising, since this factor plays a critical role in the perceived sharpness and clarity of subjective contours (Dumais \& Bradley, 1976; Parks \& Marks, 1983, 1985; Ronchi \& Mori, 1959; Warm et al., 1987). Also surprising was that increases in luminance tended to decrease the stability, rather than strengthen the visibility, of subjective contours, as was the case in the salience studies. That declines in luminance can enhance the salience of subjective contours but also undermine their stability is puzzling. Note, however, that the duration of target exposure in the salience experiments was considerably shorter $(3-5 \mathrm{sec})$ than that employed in this study $(120 \mathrm{sec})$. Perhaps the effect of fatigue that builds up over the prolonged exposure interval plays a role in modifying the influence exerted by variations in target luminance on the perceptual characteristics of subjective contours.

Recall that real contours can be rendered perceptually unstable by impoverished viewing conditions at scotopic levels (see McKinney, 1963, 1966). They can also be made unstable through the elimination of eye movements by means of stabilized retinal images (see Heckenmueller, 1965). Under such circumstances, disappearances tend to occur in an orderly fashion, obeying some general rules (MacKinnon, Forde, \& Piggins, 1969). For instance, line stimuli tend to disappear as a whole, not in parts or segments. Second, stimuli with multiple contours tend to fragment in perceptual units. For example, it is common for the two horizontal edges of a square to disappear together, or for the two vertical edges to fade away jointly.

The subjects' drawings of their remembered fragmentation experiences in this study indicate that subjective contours obey the same general rules of disappearance as do real contours. Subjects most often indicated that the forms disappeared in units, with the most typical report being one in which parallel sides of the square or diamond faded simultaneously. Single line disappearances were relatively rare, and so were reports of lines in which only segments of the line faded.
The most common form of fragmentation recalled by the subjects was whole-figure fading. In this case, the entire subjective contour disappeared, with only the inducing areas remaining visible. Complete disappearances of this sort were reported for subjective contours by Halpern and Warm (1980), who used a luminous design procedure. They have also been found in studies with real contours, but with lower frequency than in the present case (MacKinnon et al., 1969; McKinney, 1966). The greater likelihood of whole-figure disappearances with subjective contours may stem from the fact that such contours are inherently more unstable than those of real figures.

It is worth noting that our conclusions regarding qualitative similarities in the fragmentation properties of real and subjective contours are quite different from those reached by Halpern and Warm $(1980,1984)$. The earlier investigations described dramatic differences in the fragmentation characteristics of the two types of contours. Part of the disparity in results can probably be traced to the considerable difference between the scotopic illumination in the earlier studies and the primarily photopic illumination in this study. On the other hand, Halpern and Warm focused on the relative frequencies of whole-figure and contour-only disappearances and did not describe the nature of the contour fragmentation more fully. Thus, a complete comparison of their findings with those of the present study is not possible.

\section{REFERENCES}

APPElle, S. (1972). Perception and discrimination as a function of stimulus orientation: The "oblique effect" in man and animals. Psychological Bulletin, 78, 266-278.

Atkinson, J. (1972). The effect of size, retinal locus, and orientation on the visibility of a single afterimage. Perception \& Psychophysics, 12, 213-217.

Bradley, D. R., Dumais, S. T. (1984). The effects of illumination level and retinal size on the depth stratification of subjective contour figures. Perception, 13, 155-164.

Brigner, W. L., \& Gallagher, M. B. (1974). Subjective contours: Apparent depth or simultaneous brightness contrast? Perceptual \& Motor Skills, 38, 1047-1053.

Coren, S. (1972). Subjective contours and apparent depth. Psychological Review, 79, 359-367.

Dumats, S. T., \& BRADleY, D. R. (1976). The effects of illumination level and retinal size on the apparent strength of subjective contours. Perception \& Psychophysics, 19, 339-345.

Gregory, R. L. (1977). Vision and isoluminant color contrast: 1. A projection technique and observations. Perception, 6, 113-119.

HALPERN, D. F., \& W ARM, J. S. (1980). The disappearance of real and subjective contours. Perception \& Psychophysics, 28, 229-235.

HALPERN, D. F., \& WARM, J. S. (1984). The disappearance of dichoptically presented real and subjective contours. Bulletin of the Psychonomic Society, 22, 433-436.

Heckenmueller, E. G. (1965). Stabilization of the retinal image: A review of method, effects and theory. Psychological Bulletin, 63, 157-169.

JORY, M. K., \& DAY, R. H. (1979). The relationship between brightness contrast and illusory contours. Perception, 8, 3-9.

KanizsA, G. (1976, April). Subjective contours. Scientific American, 234, pp. $48-52$.

Mackinnon, G. E., Forde, J., \& Piggins, D. J. (1969). Stabilized 
images, steadily fixated figures and prolonged afterimages. Canadian Journal of Psychology, 23, 184-195.

McKInNey, J. P. (1963). Disappearance of luminous designs. Science, 140, 403-404.

MCKinney, J. P. (1966). Verbal meaning and perceptual stability. Canadian Journal of Psychology, 20, 237-242.

Parks, T. E., \& MARKS, W. (1983). Sharp-edged vs. diffuse illusory circles: The effects of varying luminance. Perception \& Psychophysics, 33, $172-176$.

Parks, T. E., \& Marks, W. (1985). Mllusory figures: Individual differences in apparent depth and lightness. Perception \& Psychophysics, 37, 529-532.

Petry, S., Harbeck, A., Conway, J., \& Levey, J. (1983). Stimulus determinants of brightness and distinctness of subjective contours. Perception \& Psychophysics, 34, 169-174.

RoNCHI, L., \& MORI, G. F. (1959). On the factors which affect the contrast enhancement in a figure with quasi perceptive contours and a practical application of such a figure. Fondazione Giorgio Ronchi Atti, 14, 495-508.
ScHuck, J. R. (1973). Factors affecting reports of fragmenting visual images. Perception \& Psychophysics, 13, 382-390.

SchumanN, F. (1987). Contributions to the analysis of visual perception-first paper: Some observations on the combination of visual impressions into units (A. Hogg, Trans.). In S. Petry \& G. E. Meyer (Eds.), The perception of illusory contours (pp. 21-33). New York: Springer-Verlag. Originally published in Zeitschrift für Psychologie und Physiologie der Sinnesorgane, 1900, 23, 1-32.

Vogels, R., \& ORBan, G. A. (1987). Illusory contour orientation discrimination. Vision Research, 27, 453-467.

Warm, J. S., Dember, W. N., Padich, R. A., Beckner, J., \& Jones, S. (1987). The role of illumination level in the strength of subjective contours. In S. Petry \& G. E. Meyer (Eds.), The perception of illusory contours (pp. 176-181). New York: Springer-Verlag.

(Manuscript received September 18, 1991; revision accepted for publication August 12, 1993.) 